EESTI NSV TEADUSTE AKADEEMIA TOIMETISED, 25. KOIDE FOOSIKA * MATEMAATIKA. 1976, NR. 2

ИЗВЕСТИЯ АКАДЕМИИ НАУК ЭСТОНСКОП ССР ТОМ 25 ФНЗНҚА * МАТЕМАТИҚА. 1976, № 2

удК $512.25 / .26+519.3: 330.1 .15$

Эбу TAMM

\title{
О КВАЗИВЫПУКЛОСТИ ФУНКЦИЙ ВЕРОЯТНОСТИ И КВАНТИЛЯ
}

Задачи стохастического программирования с целевыми функциями или ограничениями в виде функций вероятности и квантиля естественным образом возникают при решении разных экономических и технических проблем $\left[{ }^{1}\right]$ и поэтому изучение свойств этих функций представляет несомненный интерес.

Рассмотрим следующие функции вероятности и квантиля:

$$
\begin{aligned}
& v_{\gamma}(x)=P[f(x, y) \leqslant \gamma], \\
& w_{\alpha}(x)=\min \left\{z \in R^{1}, P[f(x, y) \leqslant z] \geqslant \alpha\right\}, \\
& \bar{v}_{\gamma}(x)=P[f(x, y)<\gamma], \\
& \bar{w}_{\alpha}(x)=\max \left\{z \in R^{1}, P[f(x, y)<z] \leqslant \alpha\right\},
\end{aligned}
$$

где $f(x, y)$ - действительная функция от $n$-мерного вектора $x$ и от случайного $m$-мерного вектора $y, \alpha$ и $\gamma$ - действительные числа $(0 \leqslant \alpha \leqslant 1), P$ означает вероятность. Другие возможные виды этих функций и связи между ними рассматривались в $\left[{ }^{2}\right]$.

В нелинейном программировании выпуклые и вогнутые (см., напр., $\left.\left[{ }^{3}\right]\right)$ функции имеют особое значение, поскольку в выпуклых задачах некоторые необходимые условия оптимальности (условия Куна-Такера и др.) являются и достаточными. Кроме того, для таких задач можно построить более эффективные методы решения, чем для остальных. Но во многих случаях требование выпуклости функции оказывается слишком сильным. Например, не имеет смысла требовать выпуклость функций (1) и (3) во всем пространстве, потому что они ограничены, а выпуклые и ограниченные во всем пространстве функции - постоянные. Известно (см., напр., $\left.\left[{ }^{3}\right]\right)$, что условие выпуклости в задачах математического программирования можно заменить более слабым условием квазивыпуклости *, причем упомянутые выше удобные свойства задачи сохраняются.

В данной работе показывается, какие соотношения имеют место между квазивыпуклостью и квазивогнутостью функций (1)-(4).

* Функция $h(x)$ называется в выпуклой области $X \subset R^{n}$ квазивыпуклой, если и квазивогнутой, если

$$
h\left(\lambda x_{1}+(1-\lambda) x_{2}\right) \leqslant \max \left[h\left(x_{1}\right), h\left(x_{2}\right)\right],
$$

$$
h\left(\lambda x_{1}+(1-\lambda) x_{2}\right) \geqslant \min \left[h\left(x_{1}\right), h\left(x_{2}\right)\right]
$$

для любых $x_{1}, x_{2}$ и любого $\lambda, 0 \leqslant \lambda \leqslant 1$. Если $h(x)$ квазивыпукла, то $-h(x)-$ квазивогнута. 
Т е о ре м а 1. Если $v_{\gamma}(x)$ в выпуклой области $X \subset R^{n}$ квазивыпукла (квазивогнута) для любого $\gamma$, то $w_{\alpha}(x)$ квазивогнута (квазивыпукла) в этой области для любого $\alpha$.

Д ок а з а тельст в о. Пусть $\alpha$ фиксированное, но произвольное число и пусть $v_{v}(x)$ квазивыпукла в $X$ для любого $\gamma$. Выбираем произвольно $x_{1}, x_{2} \in X$ и обозначаем

Тогда

$$
z(\lambda)=w_{\alpha}\left(\lambda x_{1}+(1-\lambda) x_{2}\right) .
$$

$$
P\left[f\left(\lambda x_{1}+(1-\lambda) x_{2}, y\right) \leqslant z(\lambda)\right] \geqslant \alpha .
$$

Из квазивыпуклости $v_{v}(x)$ для любого $\gamma$ следует

$P\left[f\left(\lambda x_{1}+(1-\lambda) x_{2}, y\right) \leqslant z(\lambda)\right] \leqslant \max \left\{P\left[f\left(x_{1}, y\right) \leqslant z(\lambda)\right] . \quad P\left[f\left(x_{2}, y\right) \leqslant z(\lambda)\right]\right\}$ и, учитывая (6), $\max \left\{P\left[f\left(x_{1}, y\right) \leqslant z(\lambda)\right], P\left[f\left(x_{9}, u\right) \leqslant z(\lambda)\right\rceil\right\} \geqslant \alpha$. Не нарушая общности. можно допустить, что $P\left[f\left(x_{1}, y\right) \leqslant z(\lambda)\right] \geqslant \alpha$. Тогда $\min \left\{z \in R^{1}, P\left[f\left(x_{1}, y\right) \leqslant z\right] \geqslant \alpha\right\} \leqslant z(\lambda), \quad$ т. е.

$$
w_{\alpha}\left(x_{1}\right) \leqslant z(\lambda) \text {. }
$$

Из (5) и (7) получаем, что $w_{\alpha}\left(\lambda x_{1}+(1-\lambda) x_{2}\right) \geqslant w_{\alpha}\left(x_{1}\right)$ и тем более $w_{\alpha}\left(\lambda x_{1}+(1-\lambda) x_{2}\right) \geqslant \min \left[w_{\alpha}\left(x_{1}\right), w_{\alpha}\left(x_{2}\right)\right]$, следовательно, $w_{\alpha}(x)$ квазивогнута.

Допустим теперь, что $v_{\gamma}(x)$ квазивогнута в $X$ для любого $\gamma$. Выбирая $\gamma=z(\lambda)-\varepsilon, \varepsilon>0$, получаем

$$
P\left[f\left(\lambda x_{1}+(1-\lambda) x_{2}, y\right) \leqslant z(\lambda)-\varepsilon\right] \geqslant
$$

$$
\geqslant \min \left\{P\left[f\left(x_{1}, y\right) \leqslant z(\lambda)-\varepsilon\right], P\left[f\left(x_{2}, y\right) \leqslant z(\lambda)-\varepsilon\right]\right\} .
$$

По определению функции квантиля (2) с учетом (5) имеем

$$
P\left[f\left(\lambda x_{1}+(1-\lambda) x_{2}, y\right) \leqslant z(\lambda)-\varepsilon\right]<\alpha .
$$

Из (8) и (9) вытекает, что $\min \left\{P\left[f\left(x_{1}, y\right) \leqslant z(\lambda)-\varepsilon\right], P\left[f\left(x_{2}, y\right) \leqslant\right.\right.$ $\leqslant z(\lambda)-\varepsilon]\}<\alpha$. Пусть $P\left[f\left(x_{1}, y\right) \leqslant z(\lambda)-\varepsilon\right]<r$, откуда $w_{\alpha}\left(x_{1}\right)>$ $>z(\lambda)-\varepsilon$. Так как $\varepsilon>0$ можно задавать сколь угодно малым, то

$$
w_{\alpha}\left(x_{1}\right) \geqslant z(\lambda) \text {. }
$$

Из (5) и (10) следует, что $w_{\alpha}\left(\lambda x_{1}+(1-\lambda) x_{2}\right) \leqslant w_{\alpha}\left(x_{1}\right)$, т. е. $w_{\alpha}(x)$ квазивыпукла. Теорема доказана.

Приведем пример, показывающий, что предположения теоремы 1 недостаточно для вогнутости функции $w_{\alpha}(x)$ для любого $\alpha$. Пусть $f(x, y)=\mathrm{e}^{-x^{2}} y$, где $x \in(-\infty, \infty), y \in[0,1]$ и распределен на этом отрезке равномерно. Функшия $f_{1}(x)=\mathrm{e}^{-x^{2}}$ квазивогнута, $f_{2}(y)=y$ неотрицательна. Согласно $\left[{ }^{2}\right], v_{\gamma}(x)=P\left[\mathrm{e}^{-x^{2}} y \leqslant \gamma\right]$ квазивыпукла для любого $\gamma$. Покажем, что $w_{1 / 2}(x)$ не является вогнутой. Выбираем $x_{1}=0, x_{2}=2 \sqrt{2}$. Тогда

$$
\begin{aligned}
w_{1 / 2}\left(x_{1}\right) & =\min \left\{z \in R^{1}, P\left[f\left(x_{1}, y\right) \leqslant z\right] \geqslant 1 / 2\right\}= \\
& =\min \left\{z \in R^{1}, P\left[\mathrm{e}^{0} \cdot y \leqslant z\right] \geqslant 1 / 2\right\}= \\
& =\min \left\{z \in R^{1}, P[y \leqslant z] \geqslant 1 / 2\right\}=1 / 2
\end{aligned}
$$

Аналогично получаем $w_{1 / 2}\left(x_{2}\right)=\frac{1}{2 \mathrm{e}^{8}}, w_{1 / 2}\left(\frac{x_{1}+x_{2}}{2}\right)=\frac{1}{2 \mathrm{e}^{2}}$ и $\frac{1}{2} w_{1 / 2}\left(x_{1}\right)+$ $+\frac{1}{2} w_{1 / 2}\left(x_{2}\right)=\frac{1}{4}+\frac{1}{4 \mathrm{e}^{8}}$. Поскольку $\frac{1}{4}+\frac{1}{4 \mathrm{e}^{8}}>\frac{1}{2 \mathrm{e}^{2}}$, то $w_{1 / 2}\left(\frac{x_{1}-1-x_{2}}{2}\right)<$ $<\frac{1}{2} w_{1 / 2}\left(x_{1}\right)+\frac{1}{2} w_{1 / 2}\left(x_{2}\right)$, и $w_{1 / 2}(x)$ действительно не является вогнутой.

Оказывается, что верно и утверждение, обратное утверждению теоремы 1 . 
Т е о рем а 2. Если $w_{\alpha}(x)$ в выпуклой области $X \subset R^{n}$ квазивыпукла (квазивогнута) для любого $\alpha$, то $v_{\gamma}(x)$ квазивогнута (квазивыпукла) в этой области для любого $\gamma$.

Д ок а з а тель с т о. Пусть $w_{\alpha}(x)$ квазивыпукла при любом $\alpha$ и $x_{1}, x_{2} \in X$. Фиксируем $\gamma$ и обозначаем

$$
\begin{array}{ll}
P\left[f\left(x_{1}, y\right) \leqslant \gamma\right]=p_{1} & \left(v_{\gamma}\left(x_{1}\right)=p_{1}\right), \\
P\left[f\left(x_{2}, y\right) \leqslant \gamma\right]=p_{2} & \left(v_{\gamma}\left(x_{2}\right)=p_{2}\right) .
\end{array}
$$

Пусть $p_{1} \geqslant p_{2}$. Тогда из определения функции квантиля (2) имеем

$$
w_{p_{1}}\left(x_{1}\right) \leqslant \gamma, \quad w_{p_{1}}\left(x_{2}\right) \leqslant \gamma .
$$

По предположению $w_{p_{1}}(x)$ квазивыпукла, т. е. $w_{p_{1}}\left(\lambda x_{1}+(1-\lambda) x_{2}\right) \leqslant$ $\leqslant \max \left[w_{p_{1}}\left(x_{1}\right), w_{p_{1}}\left(x_{2}\right)\right]$ и с учетом $(13) \max \left[w_{p_{1}}\left(x_{1}\right), w_{p_{1}}\left(x_{2}\right)\right] \leqslant \gamma$, откуда $w_{p_{1}}\left(\lambda x_{1}+(1-\lambda) x_{2}\right) \leqslant \gamma$. Из последнего неравенства вытекает, что $P\left[f\left(\lambda x_{1}+(1-\lambda) x_{2}, y\right) \leqslant \gamma\right] \geqslant p_{1}$, откуда, учитывая (11), получаем

$$
\begin{gathered}
P\left[f\left(\lambda x_{1}+(1-\lambda) x_{2}, y\right) \leqslant \gamma\right] \geqslant P\left[f\left(x_{1}, y\right) \leqslant \gamma\right], \quad \text { т. e. } \\
v_{\gamma}\left(\lambda x_{1}+(1-\lambda) x_{2}\right) \geqslant v_{\gamma}\left(x_{1}\right)
\end{gathered}
$$

и, следовательно, $v_{\gamma}(x)$ квазивогнута.

Пусть теперь $w_{\alpha}(x)$ квазивогнута для любого $\alpha$. Введем обозначение

$$
p(\lambda)=P\left[f\left(\lambda x_{1}+(1-\lambda) x_{2}, y\right) \leqslant \gamma\right] \quad\left(v_{\gamma}\left(\lambda x_{1}+(1-\lambda) x_{2}\right)=p(\lambda)\right) .
$$

Тогда $w_{p(\lambda)}\left(\lambda x_{1}+(1-\lambda) x_{2}\right) \geqslant \min \left[w_{p(\lambda)}\left(x_{1}\right), w_{p(\lambda)}\left(x_{2}\right)\right]$.

Пусть, например

$$
w_{p(\lambda)}\left(\lambda x_{1}+(1-\lambda) x_{2}\right) \geqslant w_{p(\lambda)}\left(x_{1}\right) .
$$

По (14) $w_{p(\lambda)}\left(\lambda x_{1}+(1-\lambda) x_{2}\right) \leqslant \gamma$, а в силу (15) тем более $w_{p(\lambda)}\left(x_{1}\right) \leqslant \gamma$, откуда $P\left[f\left(x_{1}, y\right) \leqslant \gamma\right] \geqslant p(\lambda) \quad\left(v_{\gamma}\left(x_{1}\right) \geqslant p(\lambda)\right)$ и, учитывая $(14), v_{\gamma}\left(\lambda x_{1}+\right.$ $\left.+(1-\lambda) x_{2}\right) \leqslant v_{\gamma}\left(x_{1}\right)$. Следовательно, $v_{\gamma}\left(\lambda x_{1}+(1-\lambda) x_{2}\right) \leqslant \max \left[v_{\gamma}\left(x_{1}\right)\right.$, $v_{\gamma}\left(x_{2}\right)$ ] и поэтому $v_{\gamma}(x)$ квазивыпукла. Теорема доказана.

Аналогичные связи имеют место и между функциями (3) и (4).

Т е оре м а 3. Если $\bar{v}_{\gamma}(x)$ в выпуклой области $X \subset R^{n}$ квазивыпукла (квазивогнута) для любого $\gamma$, то $\bar{w}_{\alpha}(x)$ квазивогнута (квазивыпукла) в этой области для любого $\alpha$.

Доказ а тельство.

$$
\begin{aligned}
\bar{v}_{\nu}(x)=P[f(x, y) & <\gamma]=1-P[f(x, y) \geqslant \gamma]=1-P[-f(x, y) \leqslant-\gamma], \\
\bar{w}_{\alpha}(x) & =\max \left\{z \in R^{1}, P[f(x, y)<z] \leqslant \alpha\right\}= \\
& =\max \left\{z \in R^{1}, 1-P[-f(x, y) \leqslant-z] \leqslant \alpha\right\}= \\
& =-\min \left\{-z \in R^{1}, 1-P[-f(x, y) \leqslant-z] \leqslant \alpha\right\}= \\
& =-\min \left\{z \in R^{1}, P[-f(x, y) \leqslant z] \geqslant 1-\alpha\right\} .
\end{aligned}
$$

Пусть $\bar{v}_{\gamma}(x)$ квазивыпукла (квазивогнута) для любого $\gamma$. Тогда в силу (16) $\hat{v}_{\gamma}(x)=P[-f(x, y) \leqslant-\gamma]$ квазивогнута (квазивыпукла) для любого $\gamma$. Из теоремы 1: $\hat{w}_{1-\alpha}(x)=\min \left\{z \in R^{1}, P[-f(x, y) \leqslant z] \geqslant\right.$ $\geqslant 1-\alpha\}$ квазивыпукла (квазивогнута) и по (17) $\bar{w}_{\alpha}(x)$ квазивогнута (квазивыпукла) для любого $\boldsymbol{\alpha}$. Теорема доказана.

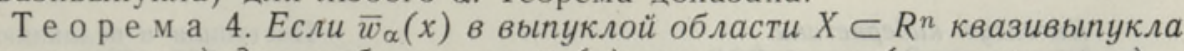
(квазивогнута) для любого $\alpha$, то $\bar{v}_{\gamma}(x)$ квазивогнута (квазивыпукла) в этой области для любого $\gamma$.

Д ок а з а те ль с т о этой теоремы аналогично доказательству теоремы 3.

Известно несколько классов функций $f(x, y)$ и распределений век- 
тора $y$, при которых функции (1) и (3) квазивыпуклы. В [ $\left.{ }^{4}\right]$ доказано, что если $f(x, y)$ вогнута в $R^{n+m}$ и распределение вектора $y \in R^{m}$ логарифмически вогнуто, т. е. $P[\lambda A+(1-\lambda) B] \geqslant\left(P[A]^{\lambda}(P[B])^{1-\lambda}\right.$ для любых выпуклых множеств $A, B \subset R^{m}$ и $\lambda \in(0,1)$, то функция $\widetilde{v}_{0}(x)=$ $=P[f(x, y) \geqslant 0]$ логарифмически вогнута в $R^{n}$. Неотрицательная функция $h(x)$, определенная на выпуклом множестве $X \subset R^{n}$, называется логарифмически вогнутой, если для любых $x_{1}, x_{2} \in X$ и $\lambda \in(0,1)$ верно $h\left(\lambda x_{1}+(1-\lambda) x_{2}\right) \geqslant\left[h\left(x_{1}\right)\right]^{\lambda}\left[h\left(x_{2}\right)\right]^{1-\lambda}$. Непосредственно вытекает, что если $h(x)$ логарифмически вогнута, то она и квазивогнута. Легко показать, что при этих условиях, наложенных на функцию $f(x, y)$ и распределение вектора $y, \widetilde{v}_{v}(x)=P[f(x, y) \geqslant \gamma]$ логарифмически вогнута для любого $\gamma$. Следовательно, $\widetilde{v}_{\gamma}(x)$ квазивогнута и $\bar{v}_{\gamma}(x)=1-\widetilde{v}_{v}(x)$ квазивыпукла. По теореме 3 при условиях $\left[{ }^{4}\right] \bar{w}_{\alpha}(x)$ квазивогнута.

В $\left[{ }^{2}\right]$ установлено, что если функция $f(x, y)$ представима в виде

$$
f(x, y)=f_{1}(x) f_{2}(y)+f_{3}(y)
$$

где $f_{1}(x)$ квазивогнута (квазивыпукла) и $f_{2}(y)$ неотрицательна (неположительна), то $v_{\gamma}(x)$ квазивыпукла для любого $\gamma$. Ниже этот результат обобщают теоремы (5) и (6).

Т е о ре м а 5. Функции $v_{\gamma}(x)$ u $\bar{v}_{\gamma}(x)$ в выпуклой области $X \subset R^{n}$ квазивыпуклы для любого $\gamma$, если $f(x, y)$ представима в виде $f(x, y)=$ $=\varphi(u(x), y)$, где $u(x)$ квазивыпукла (квазивогнута) в области $X u$ $\varphi(t, y)$ при любом у монотонно убывающая (возрастающая) функция действительного переменного.

Д ок а 3 а тел л с т в о. Пусть $f(x, y)=\varphi(u(x), y), \quad u(x)$ квазивыпукла и $\varphi(t, y)$ монотонно убывающая функция $t$ при любом $y$. Пусть, например, $u\left(\lambda x_{1}+(1-\lambda) x_{2}\right) \leqslant u\left(x_{1}\right)$. Тогда $\varphi\left(u\left(\lambda x_{1}+(1-\lambda) x_{2}, y\right) \geqslant\right.$ $\geqslant \varphi\left(u\left(x_{1}\right), y\right)$ при любом $y$. Если при каком-нибудь $\bar{y} \varphi\left(u\left(\lambda x_{1}+\right.\right.$ $\left.\left.+(1-\lambda) x_{2}\right), \bar{y}\right) \leqslant \gamma$, то и $\varphi\left(u\left(x_{1}\right), \bar{y}\right) \leqslant \gamma$. Следовательно, $P\left[\varphi\left(u\left(\lambda x_{1}+\right.\right.\right.$ $\left.\left.+(1-\lambda) x_{2}, y\right) \leqslant \gamma\right] \leqslant P\left[\varphi\left(u\left(x_{1}\right), y\right) \leqslant \gamma\right]$, т. е. $v_{\gamma}(x)$ квазивыпукла.

Остальная часть теоремы доказывается аналогично.

Т е о р е м а 6. Функции $v_{\nu}(x) \quad u \bar{v}_{\gamma}(x)$ в выпуклой области $X \subset R^{n}$ квазивогнуты для любого $\gamma$, если $f(x, y)$ представима в виде $f(x, y)=$ $=\varphi(u(x), y)$, где $u(x)$ квазивыпукла (квазивогнута) в области $X u$ $\varphi(t, y)$ при любом у монотонно возрастающая (убывающцая) функция действительного переменного.

При предположениях теоремы 6 функции (1) и (3) квазивогнуты. Доказательство с необходимыми изменениями следует доказательству теоремы 5 .

В работе над данной статьей большую помощь автору оказал Э. Райк.

\section{ЛИТЕРАТ У Р А}

1. Юдин Д. Б., Математические методы управления в условиях неполной информацин, М., 1974.

2. Р а йк Э., Изв. АН ЭССР, Физ. Матем., 21, 142 (1972).

3. З а нгвилл У. И., Нелинейное программирование. М., 1973.

4. Prék op a A., Math. Operationsforsch. u. Statist., 3, H. 5, 349 (1972).

Ннститут кибернетики Академии наук Эстонской ССР

Поступила в редакцию 7/V 1975 


\section{Ebu TAMM}

\section{TOENÄOSUS- JA KVANTIILFUNKTSIOONIDE KVAASIKUMERUSEST}

Vaadeldakse tõenäosusfunktsioone $v_{\gamma}(x)=P[f(x, y) \leqslant \gamma]$ ja $\quad \bar{v}_{\gamma}(x)=P[f(x, y)<\gamma]$ ning kvantiilfunktsioone $w_{\alpha}(x)=\min \left\{z \in R^{1}, P[f(x, y) \leqslant z] \geqslant \alpha\right\} \quad$ ja $\quad \bar{w}_{\alpha}(x)=$ $=\max \left\{z \in R^{1}, P[f(x, y)<z] \leqslant \alpha\right\}$, kus $x \in R^{n}$, juhuslik vektor $y \in R^{m}$, reaalarvud $\alpha \in[0,1]$ ja $\gamma$ on fikseeritud. Tōestatakse, et $v_{\gamma}(x)$ on kvaasikumer (kvaasinõgus) parajasti siis, kui $w_{\alpha}(x)$ on kvaasinõgus (kvaasikumer), ning et samasugune seos kehtib ka funktsioonide $\bar{v}_{\gamma}(x)$ ja $\bar{w}_{\alpha}(x)$ vahel.

On esitatud mõned funktsioonide $f(x, y)$ klassid, mille korral $v_{\gamma}(x)$ ning $\bar{v}_{\gamma}(x)$ on kvaasikumerad või kvaasinõgusad.

\section{Ebu TAMM}

\section{ON THE QUASI-CONVEXITY OF PROBABILITY AND QUANTILE FUNCTIONS}

The probability functions $v_{\gamma}(x)=P[f(x, y) \leqslant \gamma]$ and $\bar{v}_{\gamma}(x)=P[f(x, y)<\gamma]$ and quantile functions $w_{\alpha}(x)=\min \left\{z \in R^{1}, P[f(x, y) \leqslant z] \geqslant \alpha\right\}$ and $\bar{w}_{\alpha}(x)=$ $=\max \left\{z \in R^{1}, P[f(x, y)<z] \leqslant \alpha\right\}$ are considered. The vector $x \in R^{n}$, the random vector $y \in R^{m}$, and the real numbers $\alpha \in[0,1]$ and $\gamma$ are fixed.

It is shown that $v_{\gamma}(x)$ is quasi-convex (quasi-concave) if $w_{\alpha}(x)$ is quasi-concave (quasi-convex). An analogous relation also holds between $\bar{v}_{\gamma}(x)$ and $\bar{w}_{\alpha}(x)$.

Some classes of functions $f(x, y)$ are found, for which $v_{\gamma}(x)$ and $\bar{v}_{\gamma}(x)$ are quasiconvex or quasi-concave. 